\title{
Effective dielectric constants of photonic crystal of aligned anisotropic cylinders and the optical response of a periodic array of carbon nanotubes
}

\author{
E. Reyes \\ Instituto de Física, Universidad Autónoma de Puebla, Apartado Postal J-48, Puebla, 72570 Mexico \\ A. A. Krokhin and J. Roberts \\ Department of Physics, University of North Texas, P.O. Box 311427, Denton, Texas 76203, USA
}

(Received 16 May 2005; published 25 October 2005)

\begin{abstract}
We calculate the static dielectric tensor of a periodic system of aligned anisotropic dielectric cylinders. Exact analytical formula for the effective dielectric constants for the $H$-eigenmodes is obtained for arbitrary 2D Bravais lattice and arbitrary cross section of anisotropic cylinders. It is shown that depending on the symmetry of the unit cell photonic crystal of anisotropic cylinders behaves like uniaxial or biaxial natural crystals. The developed theory of homogenization of anisotropic cylinders is applied for calculations of the dielectric properties of photonic crystals of carbon nanotubes.
\end{abstract}

DOI: $10.1103 /$ PhysRevB.72.155118

PACS number(s): 42.70.Qs, 41.20.Jb, 42.25.Lc, 78.67.Ch

Periodic dielectric structures - photonic crystals (PC's) have found many useful technological applications. ${ }^{1}$ Progress in the development of optical devices that operate using the principles of the control of light ${ }^{2}$ gave rise to theoretical studies of the properties of the spectra of PC's. ${ }^{3}$ In particular, the region of low frequencies, where the methods of the theory of homogenization ${ }^{4}$ are applicable, has attracted attention in the last decade. In the low-frequency limit the light wave has linear dispersion and it is characterized by effective permittivity

$$
\boldsymbol{\epsilon}_{\mathrm{eff}}(\hat{\mathbf{k}})=\lim _{k \rightarrow 0}(c k / \omega)^{2}
$$

This effective parameter depends on the direction of propagation $\hat{\mathbf{k}}=\mathbf{k} / k$ and has tensor structure. The latter property is emphasized for 2D PC's, which are anisotropic uniaxial or biaxial crystals. ${ }^{5}$ Unlike this, 3D PC's may be isotropic. ${ }^{6}$

Optical anisotropy of the PC's studied in Refs. 5-7 is determined by the geometry of the unit cell only. The constituents themselves are considered to be isotropic dielectrics. This is not the case, for example, for a structure of aligned carbon nanotubes or $\mathrm{ZnO}$ nanorods. ${ }^{9}$ Here anisotropy manifests itself at the "microscopic" level, since the nanotubes ("atoms" of the PC) are optically anisotropic. The static values of these dielectric constants are $\varepsilon_{\|}=1.8225$ and $\varepsilon_{\perp}=5.226 .{ }^{8}$ The elongated topology and the anisotropy of graphite cause PC's of carbon nanotubes to exhibit large optical anisotropy. ${ }^{9}$ Three-dimensional PC's of anisotropic spheres have been studied in Ref. 10.

High anisotropy of 2D photonic crystals may find applications in nanophotonics as was proposed by Artigas and Torner. ${ }^{11}$ Namely, the surface of an anisotropic 2D photonic crystal supports propagation of a surface wave. ${ }^{12}$ It is localized close to the surface due to the interference between the ordinary and extraordinary waves. In natural crystals, it can be hardly observed because of the low anisotropy. Since it is a surface wave with very low energy losses, then it may replace surface plasmons in the near-field optics and integrated photonic circuits.

The exact theory presented in this paper allows a calculation of the effective dielectric constant of carbon nanotubes imbedded in a gas. Due to high absorbability of nanotubes, the concentration of gas in the interior region of the nanotubes may be different from that in the atmosphere. This leads to slightly different dielectric constants of the material in the interior and exterior regions of the cylinders. This effect can be detected measuring the shift of the resonant frequency of a cavity. ${ }^{13}$

An effective medium theory for the PC of anisotropic carbon nanotubes was proposed in Ref. 14. This theory is based on the Maxwell-Garnett approximation and it leads to a simple formula for $\varepsilon_{\text {eff }}$. The formula is valid for low filling fractions and square lattice.

In this paper we extend the results of the theory ${ }^{5}$ to the case of anisotropic dielectric cylinders. Exact formulas are obtained for the principal dielectric constants of a 2D PC with an arbitrary cross-sectional form of anisotropic cylinders, arbitrary Bravais lattice, and filling fraction. We apply our results to calculate the dielectric tensor of the $\mathrm{PC}$ of carbon nanotubes. We also consider a $\mathrm{PC}$ with a rectangular unit cell and calculate two different in-plane dielectric constants. In this case the corresponding effective medium is a biaxial crystal. It was argued that for the $H$-polarized mode the effective dielectric constant for hollow and solid cylinders are practically indistinguishable. ${ }^{14}$ Using our approach, we study the effect of the internal cavity on the effective dielectric constant and show explicitly how the effective dielectric constant decreases with the internal radius.

We consider a 2D periodic structure of dielectric cylinders with their axes parallel to $z$. The background material is an isotropic dielectric with permittivity $\varepsilon_{b}$ and the cylinders are rolled up from an anisotropic dielectric sheet characterized by a tensor $\hat{\varepsilon}^{(a)}$. For carbon nanotubes, this tensor has two different eigenvalues, and in cylindrical coordinates is represented by a diagonal matrix with elements $\varepsilon_{\theta \theta}^{(a)}=\varepsilon_{z z}^{(a)}=\varepsilon_{\perp}$ and $\varepsilon_{r r}^{(a)}=\varepsilon_{\|}$. As a whole, the periodic inhomogeneous dielectric 
medium is characterized by the coordinate-dependent blockmatrix,

$$
\hat{\varepsilon}(\mathbf{r})=\left(\begin{array}{cc}
\varepsilon_{\alpha \beta}(\mathbf{r}) & 0 \\
0 & \varepsilon_{z z}(\mathbf{r})
\end{array}\right) .
$$

Here $\varepsilon_{\alpha \beta}(\mathbf{r})$ is a $2 \times 2$ Hermitian matrix in the $x-y$ plane. Outside the cylinders it reduces to a scalar, $\varepsilon_{b} \delta_{\alpha \beta}(\alpha, \beta$ $=x, y)$ and inside the cylinders it is given by ${ }^{14}$

$$
\varepsilon_{\alpha \beta}=\left(\begin{array}{ll}
\left(x^{2} \varepsilon_{\|}+y^{2} \varepsilon_{\perp}\right) / r^{2} & \left(x y / r^{2}\right)\left(\varepsilon_{\|}-\varepsilon_{\perp}\right) \\
\left(x y / r^{2}\right)\left(\varepsilon_{\|}-\varepsilon_{\perp}\right) & \left(y^{2} \varepsilon_{\|}+x^{2} \varepsilon_{\perp}\right) / r^{2}
\end{array}\right) .
$$

The wave equations for the $E$-polarized mode depend only on the $z z$ component of the dielectric tensor Eq. (2). Therefore the effective dielectric constant in the longwavelength limit is the same as for the parallel arrangement of isotropic cylinders (not necessarily periodic),

$$
\varepsilon_{\mathrm{eff}}^{(E)}=\bar{\varepsilon}_{z z}=A_{c}^{-1} \int_{A_{c}} \varepsilon_{z z}(\mathbf{r}) d \mathbf{r} .
$$

Here $A_{c}$ is the area of the unit cell. For a binary composite $\varepsilon_{\text {eff }}^{(E)}=\bar{\varepsilon}_{z z}=f \varepsilon_{\perp}+(1-f) \varepsilon_{b}$, where $f$ is the filling fraction of the component $a$.

Anisotropy affects the $H$-polarized mode. The wave equation has the following form:

$$
\frac{\partial}{\partial x_{\alpha}}\left(a_{\alpha \beta} \frac{\partial H}{\partial x_{\beta}}\right)+\frac{\omega^{2}}{c^{2}} H=0, \quad x_{\alpha}, x_{\beta}=x, y .
$$

Here $a_{i j}$ is a $2 \times 2$ matrix with determinant 1 :

$$
a_{\alpha \beta}(\mathbf{r})=\varepsilon_{\alpha \beta}(\mathbf{r}) / \operatorname{det} \varepsilon_{\alpha \beta}(\mathbf{r}) .
$$

To obtain the long-wavelength limit for Eq. (5) we apply the method of plane waves. ${ }^{5}$ Using the Bloch theorem we get the Fourier expansions,

$$
H(\mathbf{r})=\exp (i \mathbf{k} \cdot \mathbf{r}) \sum_{\mathbf{G}} h_{\mathbf{k}}(\mathbf{G}) \exp (i \mathbf{G} \cdot \mathbf{r}),
$$

where $\mathbf{G}$ are the reciprocal-lattice vectors. Substituting Eq. (7) into Eq. (5) we get,

$$
\sum_{\mathbf{G}^{\prime}} a_{\alpha \beta}\left(\mathbf{G}-\mathbf{G}^{\prime}\right)(k+G)_{\alpha}\left(k+G^{\prime}\right)_{\beta} h_{\mathbf{k}}\left(\mathbf{G}^{\prime}\right)=(\omega / c)^{2} h_{\mathbf{k}}(\mathbf{G}) .
$$

Here $a_{\alpha \beta}(\mathbf{G})$ is the Fourier component of $a_{\alpha \beta}(\mathbf{r})$. The homogenization occurs if the Bloch wave (7) approaches a plane wave, i.e., if the Fourier coefficients with $G \neq 0$ in (7) vanish in the long-wavelength limit. To obtain the behavior of $h_{\mathbf{k}}(\mathbf{G})$ we substitute $G=0$ in Eq. (8) and take the limit $k$ $\rightarrow 0$,

$$
\omega^{2} / c^{2}-\bar{a}_{\alpha \beta} k_{\alpha} k_{\beta}=\sum_{\mathbf{G}^{\prime} \neq \mathbf{0}} a_{\alpha \beta}\left(-\mathbf{G}^{\prime}\right) k_{\alpha} G_{\beta}^{\prime} h_{\mathbf{k}}^{\star}\left(\mathbf{G}^{\prime}\right) .
$$

Here $\bar{a}_{\alpha \beta} \equiv a_{\alpha \beta}(\mathbf{G}=0)$ is the bulk average of the matrix (6) and $h_{\mathbf{k}}^{\star}(\mathbf{G})=h_{\mathbf{k}}(\mathbf{G}) / h_{\mathbf{k}}(\mathbf{G}=0)$. In the long-wavelength limit the coefficients of $h_{\mathbf{k}}^{\star}\left(\mathbf{G}^{\prime}\right)$ on the right-hand side are proportional to $k$. In order to make the rhs quadratic with $k$, the amplitudes of nonzero harmonics, $h_{\mathbf{k}}^{\star}\left(\mathbf{G}^{\prime}\right)$ must be propor- tional to $k$. Thus, the Bloch wave (7) can be written as a linear expansion over $k$,

$$
H(\mathbf{r})=\exp (i \mathbf{k} \cdot \mathbf{r}) h_{0}\left[1+\sum_{\mathbf{G} \neq 0} h_{\mathbf{k}}^{\star}(\mathbf{G}) \exp (i \mathbf{G} \cdot \mathbf{r})\right] .
$$

Since the sum over $\mathbf{G}$ vanishes linearly with $k$, Eq. (10) shows that the medium becomes homogeneous, i.e., the solution of the wave equation (5) approaches a plane. Now, to calculate the dielectric constant (1), we develop a perturbation theory with respect to a small parameter $k a$ ( $a$ is the lattice period). In Eq. (8) we keep the linear terms and obtain the following relation:

$$
a_{\alpha \beta}(\mathbf{G}) G_{\alpha} k_{\beta}=-\sum_{\mathbf{G}^{\prime} \neq \mathbf{0}} a_{\alpha \beta}\left(\mathbf{G}-\mathbf{G}^{\prime}\right) G_{\alpha} G_{\beta}^{\prime} h_{\mathbf{k}}^{\star}\left(\mathbf{G}^{\prime}\right) .
$$

The quadratic approximation is given by Eq. (9), which gives another linear relation between the eigenvectors $h_{\mathbf{k}}^{\star}(\mathbf{G})$. The linear relations, Eqs. (9) and (11), are the homogenized equations for the Fourier components of the magnetic field. These equations are consistent, if the corresponding determinant vanishes. Omitting the details, which can be found in Ref. 5, we give the final answer for the inverse effective dielectric constant

$$
\begin{aligned}
1 / \varepsilon_{\mathrm{eff}}^{(H)}(\hat{\mathbf{n}})= & \bar{a}_{\alpha \beta} n_{\alpha} n_{\beta}-\sum_{\mathbf{G}, \mathbf{G}^{\prime} \neq \mathbf{0}} a_{\alpha \beta}(\mathbf{G}) a_{\gamma \delta}\left(-\mathbf{G}^{\prime}\right) \\
& \times n_{\beta} G_{\alpha} n_{\gamma} G_{\delta}^{\prime}\left[a_{\lambda \mu}\left(\mathbf{G}^{\prime}-\mathbf{G}\right) G_{\mu} G_{\lambda}^{\prime}\right]^{-1} .
\end{aligned}
$$

Here $\mathbf{n}=\mathbf{k} / k$ is the unit vector in the direction of propagation and $[\cdots]^{-1}$ implies the inverse matrix in $\mathbf{G}$-space. Equation (12) is valid for an arbitrary form of the unit cell, geometry of the cylindrical inclusions, material composition of the photonic crystal, and the direction of propagation in the plane of periodicity. In the case when $a_{\alpha \beta}(\mathbf{G})=\eta(\mathbf{G}) \delta_{\alpha \beta}$ Eq. (12) is reduced to the formula obtained for isotropic cylinders in Ref. 5 [ $\eta(\mathbf{G})$ is the Fourier component of the inverse dielectric constant]. As any natural crystal, artificial PC in the long-wavelength limit can be characterized by an index ellipsoid. ${ }^{15}$ In the $x-y$ plane the cross section of the index ellipsoid is given by Eq. (12) and the $z$ semiaxis is given by Eq. (4). If the unit cell possesses a third-or higher-order rotational axis $z$, then the ellipse is reduced to a circle. In this case PC behaves like a uniaxial crystal; otherwise, it is biaxial. $^{15}$

First we study 2D PC of solid carbon cylinders arranged in square and rectangular lattices. In Cartesian coordinates the dielectric function of a carbon cylinder is given by Eq. (3). For rectangular and square lattices with circular cylinders the semiaxes of the index ellipsoid are directed along the basic lattice vectors. Because of the cylindrical symmetry of the inclusions, the off-diagonal elements of the tensor $a_{i k}(\mathbf{G})$ vanish. ${ }^{16}$ The diagonal elements for hollow cylinders with outer and inner radii $R$ and $\gamma R$, respectively, $(0 \leqslant \gamma$ $\leqslant 1$ ) have the following form:

$$
a_{x x}(0)=\varepsilon_{b}^{-1}+\frac{\pi R^{2}\left(1-\gamma^{2}\right)}{2 A_{c}}\left(\varepsilon_{\|}^{-1}+\varepsilon_{\perp}^{-1}-2 \varepsilon_{b}^{-1}\right),
$$




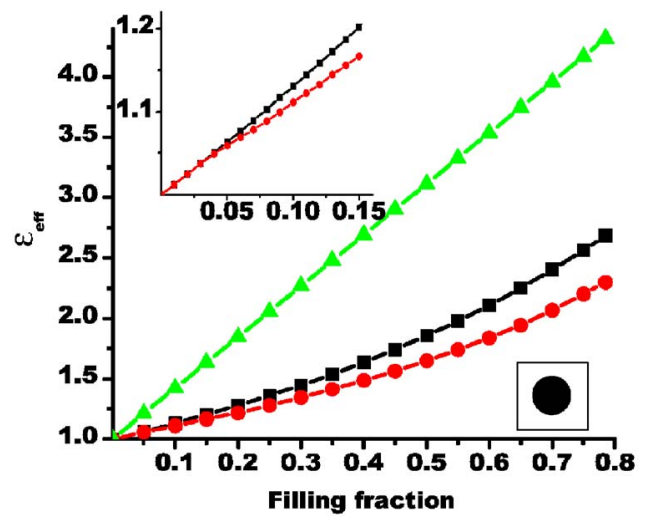

FIG. 1. (Color online) In-plane effective dielectric constant for the $H$-mode for uniaxial PC of solid graphite cylinders (circles). Straight line (triangles) is the effective dielectric constant for the $E$-mode. The squares show the results of the Maxwell-Garnett approximation (14). Insert shows the region of small filling fractions.

$$
\begin{aligned}
a_{x x}(\mathbf{G})= & 2 \pi /\left(A_{c} G^{2}\right)\left\{G R\left(\varepsilon_{b}^{-1}-\varepsilon_{\perp}^{-1}\right)\left[\gamma J_{1}(\gamma G R)-J_{1}(G R)\right]\right. \\
& \left.+\left(\varepsilon_{\|}^{-1}-\varepsilon_{\perp}^{-1}\right)\left[J_{0}(G R)-J_{0}(\gamma G R)\right]\right\} .
\end{aligned}
$$

The diagonal element $a_{y y}(\mathbf{G})$ is obtained from Eq. (13) by the replacement $\varepsilon_{\perp} \leftrightarrow \varepsilon_{\|}$.

For solid cylinders $\gamma \equiv 0$. The circles in Fig. 1 show the effective dielectric constant of the $H$-mode as a function of the filling fraction, $f=\pi R^{2} / A_{c}$, for the uniaxial PC with a square lattice. The number of plane waves considered in this calculation was 1200 , which provides a good convergence in Eq. (12). The dielectric constant for the extraordinary mode (E-mode), Eq. (4) (shown by triangles in Fig. 1) is always larger than that for the ordinary wave ( $H$-mode). Therefore, the effective medium is a uniaxial positive optically anisotropic crystal. To check the validity of the Maxwell-Garnett approximation, we plot in Fig. 1 (squares) the effective dielectric constant proposed in Ref. 14,

$$
\varepsilon_{\mathrm{MG}}^{(H)}=\frac{\varepsilon_{\|}+\Delta+f\left(\varepsilon_{\|}-\Delta\right)}{\varepsilon_{\|}+\Delta-f\left(\varepsilon_{\|}-\Delta\right)} .
$$

Here $\Delta=\sqrt{\varepsilon_{\|} / \varepsilon_{\perp}}$. One can see that for all filling fractions the Maxwell-Garnett approximation gives overestimated values for the effective dielectric constant. For a very dilute system, $f<0.07$, the Maxwell-Garnett approximation gives results that are practically indistinguishable from the exact ones (see insert in Fig. 1). For the close-packed array of cylinders the Maxwell-Garnet approximation overestimates the dielectric constant by about $25 \%$. In Fig. 2 we plot the two principal dielectric constants for the biaxial PC of solid carbon cylinders with a rectangular unit cell. The ratio of the sides of the rectangle is 1:2. The difference between the two dielectric constants increases with the filling fraction, giving rise to a higher anisotropy of the corresponding effective medium. The Maxwell-Garnett approximation Eq. (14), which does not take into account the anisotropy of the unit cell, gives the values for $\varepsilon_{\mathrm{MG}}$ that lie between the two principal values, $\varepsilon_{1}$ $<\varepsilon_{\mathrm{MG}}<\varepsilon_{2}$.

In our model we consider the carbon nanotubes as hollow graphite cylinders. In the experimental study ${ }^{9}$ of the dielec-

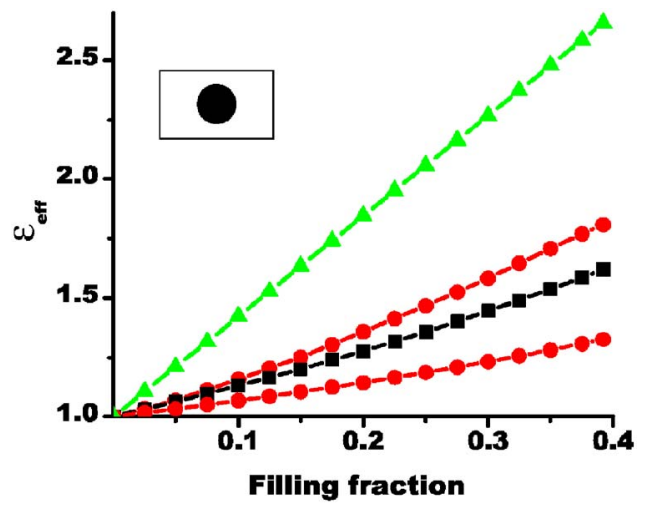

FIG. 2. (Color online) A plot of the principal effective dielectric constants for the PC of solid carbon cylinders arranged in a rectangular lattice. The larger (smaller) dielectric constant $\varepsilon_{1}\left(\varepsilon_{2}\right)$ corresponds to the direction of the vector $\mathbf{E}$ along the short (long) side of the rectangle (circles). The Maxwell-Garnett dielectric constant is shown by the squares.

tric properties of carbon nanotubes the outer radius of the cylinders was approximately $R=5 \mathrm{~nm}$. The nanotubes formed a thin film and they were oriented along a specific direction. Although the nanotubes were not necessarily arranged periodically, one can assume that they formed almost a regular lattice, since the nanotube density is about $0.6-0.7$ which is near the value of $f_{c}=\pi / 4 \approx 0.785$ for a closepacked structure. Thus, the separation between the nanotubes slightly exceeds $2 R$, and in Ref. 14 it was estimated to be $d=10.15 \mathrm{~nm}$. The inner radius is in the range $\gamma R$ $=0.25-2 \mathrm{~nm} .{ }^{14}$ The parameters $f, R, \gamma$, and $A_{c}$ are not independent but related by the formula, $f=\pi R^{2}\left(1-\gamma^{2}\right) / A_{c}$. Substitution of these parameters into this formula shows that they are self-consistent. It is worthwhile to mention that the background material in the experiment ${ }^{9}$ is not air but the host material Delrin or Teflon with $\varepsilon_{b}>1$. Since neither the density of the host material nor its dielectric constant is known, one cannot expect very good agreement between the experimental results ${ }^{9}$ and theory. In all theoretical considerations it was assumed that $\varepsilon_{b}=1$. Because of this lack of data, the effective medium theories ${ }^{14,17}$ and the results shown in Fig. 1 give lower values for $\varepsilon_{\text {eff }}$ than that observed in the experiment. ${ }^{9}$

It is obvious that the inner cavity reduces the permittivity of an isolated nanotube as compared to a solid graphite cylinder. It was argued ${ }^{14}$ that the effect of the inner cavity is less than that for a single cylinder and even can be ignored, if $\gamma \leqslant 0.4$. This conclusion was supported by comparing the results of the Maxwell-Garnet approximation Eq. (14) and numerical band structure calculations. In Fig. 3 we plot the dielectric constant for a square lattice of hollow carbon nanotubes and compare the exact results obtained from Eqs. (12) and (13) with the results given by the Maxwell-Garnett approximation. One can see that, for the same outer radius, the effective dielectric constant drops with an increase of the inner radius. Thus, if the outer radius is fixed, the dependence on the inner radius cannot be ignored, even in the Maxwell-Garnett approximation. However, the dielectric constant is much less sensitive to the internal radius if it is plotted against filling fraction, Fig. 4. 


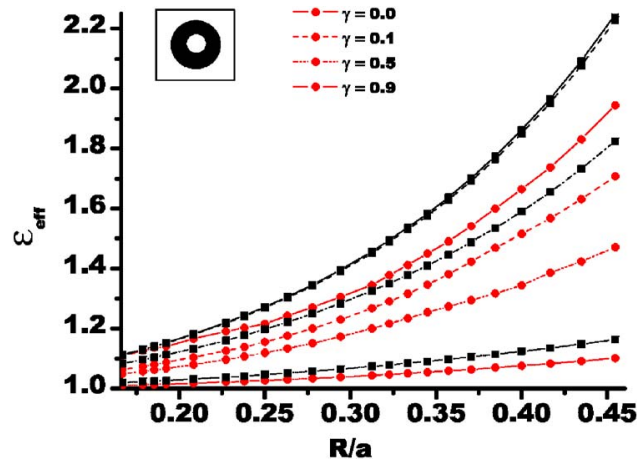

FIG. 3. (Color online) The plot of the effective dielectric constant for the square lattice of carbon nanotubes versus the outer radius for tubes with different ratios of the inner and outer radii, $\gamma=0.1,0.3,0.5,0.7$. The exact results are shown by circles and the Maxwell-Garnett approximation is shown by squares.

In the Maxwell-Garnet approximation (14) there is no dependence on the parameter $\gamma$, therefore, this approximation is represented by a single curve in Fig. 4. Here, only the filling fraction $f$ is important, but not the topology of the cylinders. In the exact theory the effective dielectric constant depends on the details of the microstructure, but as far as the filling fraction is concerned, the topology plays a much less important role. Since the cylinder is determined by either two parameters out of $R, \gamma$, and $f$, the curves in Fig. 4 may cross each other.

We calculated the static dielectric tensor for the 2D photonic crystal of anisotropic parallel cylinders arranged in a periodic lattice. The results are applied for the periodic arrangement of carbon nanotubes which are rolled up from a uniaxial graphite crystal. It was shown that the geometry of the unit cell has a small effect on the dielectric properties of

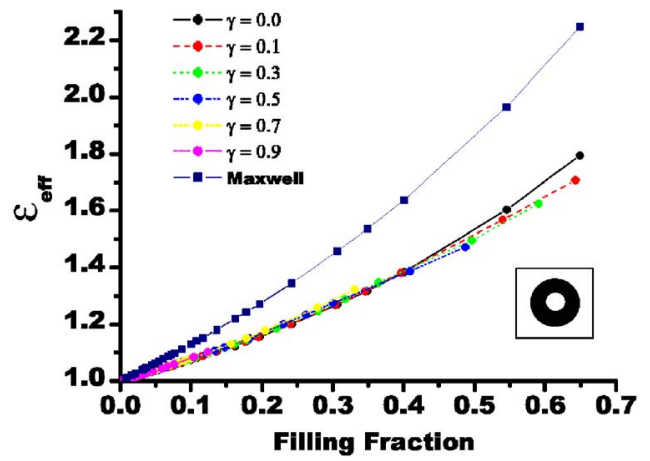

FIG. 4. (Color online). A graph of the effective dielectric constant for a square lattice of carbon nanotubes versus filling fraction for tubes with different ratios of the inner and outer radii, $\gamma$ $=0.0,0.1,0.3,0.5,0.7,0.9$. The exact results are shown by circles and the Maxwell-Garnett approximation is shown by squares.

the uniaxial PC, provided that the filling factor is fixed (see Fig. 4). Although we are interested in the static dielectric tensor, it is clear that the developed long-wavelength limit approach remains valid, even for optical frequencies since the period of the lattice of carbon nanotubes $d=10 \mathrm{~nm}$ is much less than the optical wavelength $\lambda \approx 500 \mathrm{~nm}$. To calculate the dynamic dielectric tensor, one has to substitute in the general formula Eq. (12), the corresponding frequencydependent values for $\varepsilon_{\|}$and $\varepsilon_{\perp}$. At finite frequencies Eq. (12) gives the real part of the dielectric function. For dielectrics the imaginary part of $\epsilon_{\text {eff }}$ appears in the next approximation over $\omega$. For metals Eq. (12) in invalid since $\operatorname{Re} \varepsilon^{(a)} \propto 1 / \omega$ $\rightarrow \infty$. Calculations for metallic cylinders require a generalization of the presented theory.

This work is supported by CONACyT (Mexico) Grant No. 42136-F.
${ }^{1}$ Roadmap to Photonic Crystals, edited by S. Noda and T. Baba (Kluwer, Boston, 2003).

${ }^{2}$ E. Yablonovitch, Phys. Rev. Lett. 58, 2059 (1987).

${ }^{3}$ P. Kuchment, "The Mathematics of Photonic Crystals," in Mathematical Modeling in Optical Science, edited by G. Bao, L. Cowsar, and Wen Masters (SIAM, Phildelphia, 2001), Vol. 22, p. 207; M. Birman and T. Suslina, Operator Theory Adv. Appl. 129, 71 (2001).

${ }^{4}$ M. Sahimi, Heterogeneous Materials (Springer, New York, 2003).

${ }^{5}$ P. Halevi, A. A. Krokhin, and J. Arriaga, Phys. Rev. Lett. 82, 719 (1999); A. A. Krokhin, P. Halevi, and J. Arriaga, Phys. Rev. B 65, 115208 (2002).

${ }^{6}$ S. Datta, C. T. Chan, K. M. Ho, and C. M. Soukovlis, Phys. Rev. B 48, 14936 (1993).

${ }^{7}$ A. A. Krokhin and E. Reyes, Phys. Rev. Lett. 93, 023904 (2004).

${ }^{8}$ Handbook of Optical Constant of Solids, edited by E. D. Palik (Academic, Orlando, 1991).

${ }^{9}$ W. A. de Heer, W. S. Bacsa, A. Châtelain, T. Gerfin, R. Humphrey-Baker, L. Forro, and D. Ugarte, Science 268, 845 (1995); F. Bommeli, L. Degiorgi, P. Wachter, W. S. Bacsa, W. A. de Heer, and L. Forro, Solid State Commun. 99, 513 (1996);
P. Wu, B. Kimball, J. Carlson, and D. V. G. L. N. Rao, Phys. Rev. Lett. 93, 013902 (2004); K. Kempa, B. Kimball, J. Rybczynski, Z. P. Huang, P. F. Wu, D. Steeves, M. Sennett, M. Giersig, D. V. G. L. N. Rao, D. L. Carnahan, D. Z. Wang, J. Y. Lao, W. Z. Li, and Z. F. Ren, Nano Lett. 3, 13 (2003).

${ }^{10}$ I. H. H. Zabel and D. Stroud, Phys. Rev. B 48, 5004 (1993); Zh.-Yu. Li, J. Wang, and B.-Yu. Gu, ibid. 58, 3721 (1998).

${ }^{11}$ D. Artigas and L. Torner, Phys. Rev. Lett. 94, 013901 (2005).

${ }^{12}$ M. I. Dyakonov, Sov. Phys. JETP 67, 714 (1988).

${ }^{13}$ A. Anand, J. A. Roberts, F. Naab, J. N. Dahiya, O. W. Holland, and F. D. McDaniel, Select Gas Absorption in Carbon Nanotubes Loading a Resonant Cavity to Sence Airborne Toxins Gases, Nuclear Instruments and Methods B (Elsivier, New York, 2005).

${ }^{14}$ F. J. García-Vidal, J. M Pitarke, and J. B. Pendry, Phys. Rev. Lett. 78, 4289 (1997).

${ }^{15}$ L. D. Landau, E. M. Lifshitz, and L. P. Pitaevskii, Electrodynamics of Continuous Media, 2nd ed. (Pergamon, Oxford, 1984).

${ }^{16}$ The off-diagonal elements are proportional to the integral $\oint \sin \theta \cos \theta \exp (-i G r \cos \theta) d \theta$, which vanishes identically.

${ }^{17}$ W. Lü, J. Dong, and Z.-Y. Li, Phys. Rev. B 63, 033401 (2000). 